Int. J. Odontostomat., 10(2):255-260, 2016

\title{
Periimplantitis: Revisión de la Literatura
}

\author{
Peri-Implantitis: Literature Review
}

\begin{abstract}
Alma Graciela García-Calderón*; Alejandro Donohue-Cornejo*; María Verónica Cuevas-González ${ }^{* *}$; Roberto Ávila-Valdéz ${ }^{\star \star * *} \&$ Juan Carlos Cuevas-González ${ }^{\star \star * \star *}$
\end{abstract}

GARCÍA-CALDERÓN, A. G.; DONOHUE-CORNEJO, A.; CUEVAS-GONZÁLEZ, M. V.; ÁVILA-VALDÉZ, R. \& CUEVASGONZÁLEZ, J. C. Periimplantitis: revisión de la literatura. Int. J. Odontostomat., 10(2):255-260, 2016.

RESUMEN: El uso de implantes ha tomado un gran auge en los últimos años, sin embargo así como se ha visto un aumento en la demanda también se ha visto un incremento en el fracaso de los mismos, existen múltiples razones que intervienen para esto como son; deficiencias en la cantidad y calidad de hueso, patologías óseas preexistentes, mala técnica quirúrgica, implante inadecuado, hábito tabáquico, entre otras. La mucositis periimplantaria y la periimplantitis son las primeras causas de complicaciones en los implantes, los pacientes con enfermedades crónico degenerativas, fumadores y con mala higiene son considerados pacientes de riesgo para presentar periimplantitis. La literatura ofrece tratamientos quirúrgicos y no quirúrgicos los cuales van enfocados a la eliminación de microorganismos y a la desinfección de la superficie del implante, el tratamiento quirúrgico como la debridación y curetaje tienen la finalidad de eliminar la placa bacteriana, desinfectar la superficie del implante así como retirar el tejido dañado por el proceso inflamatorio, el realizar una adecuada historia clínica y valoración previa, permitirá identificar a los pacientes con mayor o menor riesgo de desarrollar periimplantitis, las citas de mantenimiento ayudan a detectar oportunamente posibles complicaciones.

PALABRAS CLAVE: periimplantitis; mucositis periimplantaria; enfermedad periodontal.

\section{INTRODUCCION}

El uso de implantes ha tomado un gran auge en los últimos años, sin embargo así como se ha visto un aumento en la demanda también se ha visto un incremento en el fracaso de los mismos, existen múltiples razones que intervienen para esto como son; deficiencias en la cantidad y calidad de hueso, patologías óseas preexistentes, mala técnica quirúrgica, implante inadecuado, hábito tabáquico, entre otras, sin embargo a pesar de controlar en medida de lo posible los factores antes mencionados, se llega a presentar el fracaso en el sitio de colocación del implante, dentro de las principales causas se encuentra la periimplantitis, la cual en términos clínicos se define como un proceso inflamatorio que afecta la función fisiológica del tejido duro y blando que se encuentra alrededor de un implante osteointegrado, o en vías de la osteointegración (McCrea, 2014) (Fig. 1) y la mucositis periimplantaria, en donde se aprecia una inflamación reversible del tejido circundante al implante, sin que haya perdida de tejido óseo.

La prevalencia que se conoce sobre el desarrollo de la periimplantitis es muy variada, Schmidlin et al. (2012) realizaron un estudio transversal dirigido a odontólogos en donde determinaron una prevalencia que entre del $5-6 \%$ y $7-9 \%$ posterior a 5 y 10 años

Cirujana Dentista, Especialista en Periodoncia, Departamento de Estomatología, Instituto de Ciencias Biomédicas, Universidad Autónoma de Ciudad Juárez, Ciudad Juárez, Chihuahua, México.

* Cirujano Dentista, Especialista en Patología Bucal, Departamento de Estomatología, Instituto de Ciencia Biomédicas, Universidad Autónoma de Ciudad Juárez, Ciudad Juárez, Chihuahua, México.

*** Cirujana Dentista Especialista en Prótesis Maxilofacial, Alumna de la Maestría en Ciencias Estomatológicas, Facultad de Odontología, Universidad Juárez del Estado de Durango, Durango, México.

**** Cirujano Dentista Especialista en Prótesis Bucal Fija y Removible y en Periodoncia, Profesor Investigador, Facultad de Odontología, Universidad Juárez del Estado de Durango. Durango, México.

****** Cirujano Dentista, Especialista en Patología Bucal, Departamento de Estomatología, Instituto de Ciencias Biomédicas, Universidad Autónoma de ciudad de Juárez, Ciudad Juárez, México. 


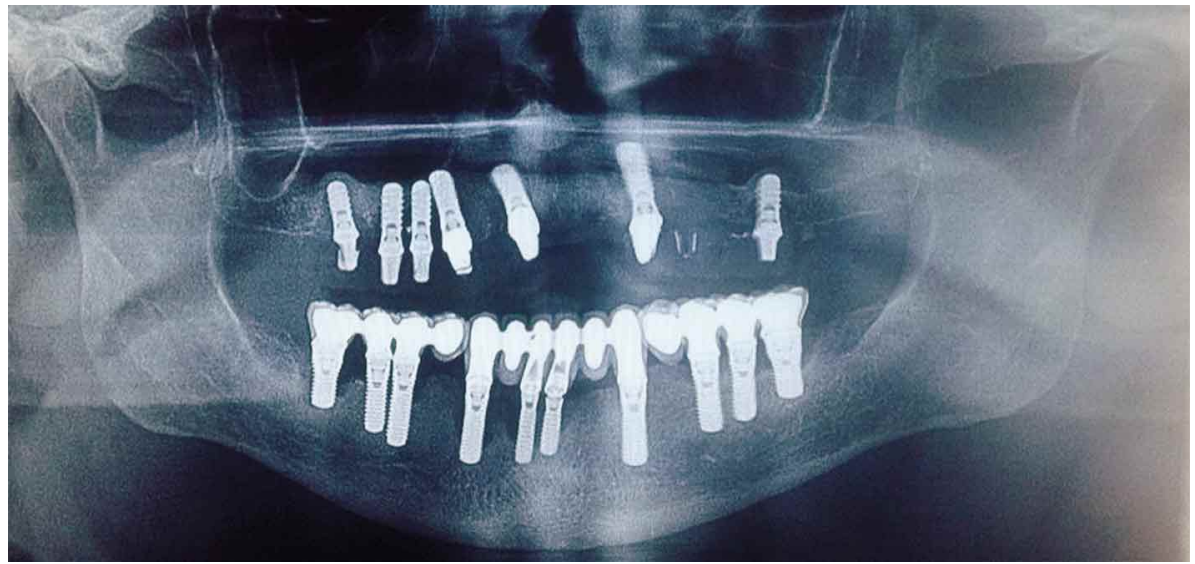

Fig. 1. Radiografía panorámica en la cual se observan defectos óseos marginales a los implantes.

respectivamente de la colocación del implante. Cavalli et al. (2015) en un estudio retrospectivo incluyeron 69 pacientes y colocaron 336 implantes y encontraron que la periimplantitis se presentaba con una prevalencia menor al 4,5\% atribuida al paciente y menor al 3,5\% atañida al implante.

Real-Osuna et al. (2012) indicaron que la mucositis periimplantaria y la periimplantitis son las primeras causas de complicaciones en los implantes con un 24 y 13,7\% respectivamente. Kadkhodazadeh \& Amid (2013) propusieron una nueva clasificación para la enfermedad periimplantaria en la cual se especifica el origen de estas patologías en conjunto con la enfermedad periodontal:

- Lesiones periodontales primarias. En la cual el origen de las lesiones es en el periodonto de los dientes adyacentes al implante y la infección se puede presentar apical, marginal y apical en el implante.

- Complicaciones primarias del implante. Aquí se describen las lesiones que se presentan debido al control inadecuado de placa bacteriana.

- Lesiones periodontales y periapicales que ocurren de forma simultanea pero separadas la una de la otra. El intervalo de tiempo entre el desarrollo de la lesión en un diente y en el implante indica que las dos lesiones se han originado por situaciones diferentes, pero han coincidido.

Etiología. Dentro de las principales causas para el desarrollo de la periimplantitis se encuentra la enfermedad periodontal preexistente, la acumulación de placa no necesariamente conlleva a la prolongación del proceso inflamatorio, sin embargo en el implante es diferente, debido a que el infiltrado inflamatorio en la mucosa periimplantaria presenta una extensión periapical más marcada. Otro factor a considerar es la presencia de tejido queratinizado alrededor de los implantes, ya que aquellos que se encuentran rodeados de encía libre no queratinizada tienen un mayor riesgo de desarrollar periimplantitis, (García-Calderón et al., 2004) la unión gingivodentaria es totalmente diferente a la unión del tejido conectivo con la superficie del implante, en este ultimo la unión de las fibras es principalmente longitudinal a diferencia de la unión gingivodentaria en donde es perpendicular, lo cual crea una entrada en línea recta a los microorganismos, facilitando la destrucción del tejido de soporte del implante (Sánchez-Garcés \& Gay-Escoda, 2004).

Casado et al. (2013) realizaron un estudio en el cual incluyeron 215 pacientes con edentulismo parcial y que contaban con implantes osteointegrados, evaluaron el riesgo de desarrollar periimplantitis relacionada a la presencia de periodontitis crónica, obteniendo como resultado que aquellos pacientes con enfermedad periodontal crónica tenían 4 veces mayor riesgo de desarrollar periimplantitis. Maruyama et al. (2014) reportaron que la periimplantitis se desarrollaba a partir de microorganismos provenientes de los órganos dentarios remanentes, sin embargo los microorganismos varían en un mismo órgano dentario y en el implante del mismo individuo, esto es debido a la presencia de ligamento periodontal y de la vascularidad en el periodonto. Dentro de los principales microorganismos relacionados a la periimplantitis se encuentran el Aggregatibacter actinomycetemcomitans, Porphyromona gingivalis, Treponema denticola, Tannerella forsythia y la Prevotella interme- 
dia si bien estos se encuentran tanto en periodonto sano como en periodontopatias, la presencia que se observa de estos microorganismos es más elevada en la periimplantitis, esto de acuerdo a Ata-Ali et al. (2011). Dahir (2013) menciono que el Staphylococcus aureus tiene una gran afinidad por la superficie de titanio de los implantes.

Como ya se había mencionado otro factor importante a considerar es la presencia de enfermedades sistémicas como diabetes, estados de inmunosupresión, tratamientos oncológicos etc. los pacientes con enfermedades crónico degenerativas como la diabetes pobremente controlada son considerados pacientes de riesgo de presentar periimplantitis, y esto es debido a que tienen una respuesta inmune así como un proceso de cicatrización pobre (Segura Andrés et al., 2015), esto lo confirma Ferreira et al. (2006) al indicar que la periodontitis y la diabetes aumentaban el riesgo de periimplantitis.

Contar con historia de tabaquismo es otro factor que puede provocar daño a los tejidos periimplantarios, ya que se conoce que la nicotina daña la síntesis de proteínas y afecta la capacidad de adhesión de los fibroblastos de la encía, lo que conlleva a alteraciones en el mantenimiento y remodelado del tejido conectivo (Baig \& Rajan, 2007), si a lo anterior se le suma una mala higiene que favorezca la formación de la placa bacteriana y la entrada de microorganismos a la zona de colocación del implante, aumenta considerablemente el riesgo del desarrollo de periimplantitis. Kasat \& Ladda (2012) realizaron una revisión acerca del daño del tabaco en el cual indicaron que la tasa de fracaso de los implantes en fumadores va del 6,5 al $20 \%$.

La periimplantitis retrograda es otra de las enfermedades periimplantarias la cual se describe radiográficamente como una lesión radiolúcida en la zona apical del implante (Rodríguez-Ortega et al., 2013). Se ha sugerido que el origen de esta lesión es a partir de microorganismos presentes en el alveolo durante la cicatrización o bien por lesiones periapicales en dientes adyacentes, de igual forma se ha sugerido que los microorganismos pueden reactivarse en el procedimiento quirúrgico durante la colocación del implante (Mohamed et al., 2012), por lo que se hace obligatorio el realizar un examen clínico minucioso que lleve a detectar los futuros focos de infección y así evitar el desarrollo de la periimplantitis retrograda o apical.

Diagnóstico. Las probabilidades de que un implante dental fracase se aumentan cuando hay una pérdida ósea mayor a $1 \mathrm{~mm}$ dentro del primer año y mayor de $2 \mathrm{~mm}$ después del primer año (Prathapachandran \& Suresh, 2012), la profundidad al sondeo que por lo general se presenta es alrededor de $3 \mathrm{~mm}$, sin embargo esta puede variar dependiendo del tipo de implante o bien por la localización submucosa del conector, el sondeo mayor a $4 \mathrm{~mm}$ puede indicar la presencia de inflamación del tejido periimplantario (García-Calderón et al.).

Para que haya un estado de salud favorable periodontal y periimplantario se requiere de un equilibrio entre las células del sistema inmune; las citocinas como bien se sabe son reguladores celulares, las cuales son producidas por los linfocitos $\mathrm{T}$ y los monocitos en presencia de infiltrado inflamatorio, las principales que se han relacionado con la destrucción de tejido periodontal son la interleucina 1-Beta (IL 1-B) y la interleucina 6 (IL-6). Yaghobee et al. (2014) reportaron que en el líquido crevicular alrededor de los implantes con periimplantitis es mayor la concentración de IL 1B que en los implantes sanos. De acuerdo a Candel-Marti et al. (2011) con el desarrollo de la periimplantitis se ha visto un aumento de las interleucinas 6,10 y 12 .

Tratamiento. La literatura ofrece tratamientos quirúrgicos y no quirúrgicos los cuales van enfocados a la eliminación de microorganismos y a la desinfección de la superficie del implante, Smeets et al. (2014) indicaron el uso local y sistémico de la tetraciclina, doxiciclina, ciprofloxacino y sulfonamidas más trimetoprima, esto al lograr reducción de la profundidad de las bolsas en un periodo de seguimiento entre uno y seis años, sin embargo recomiendan que su uso se lleve a cabo en combinación con otras técnicas manuales.

El tratamiento quirúrgico como la debridación y curetaje tienen la finalidad de eliminar la placa bacteriana, desinfectar la superficie del implante, así como retirar el tejido dañado por el proceso inflamatorio (Taschieri et al., 2015), este procedimiento se reserva para estadios avanzados de la periimplantitis en donde existe una marcada destrucción ósea.

El uso de antisépticos para la eliminación de bacterias que se depositan en el nicho periimplantar es de gran utilidad para aumentar el éxito del tratamiento de la periimplantitis, Pedrazzi et al. (2014) realizaron una revisión acerca del uso de antisépticos en el cual mencionaron que los mejores efectos en la eli- 
minación de microorganismos son los que se realizan a base de clorhexidina al $0,12 \%$ y de aceites esenciales como timol, eucalipto, mentol y salicilato de metilo, entre otros (Pedrazzi et al.).

Valderrama et al. (2014) realizaron una revisión acerca de los tratamientos no quirúrgicos en el cual mencionaron que la irrigación subgingival con clorhexidina al 0,12\% suprime los microorganismos alrededor de tres meses, sin embargo Muthukuru et al. (2012) publicaron una revisión en la cual evaluaron el tratamiento no quirúrgico para la periimplantitis concluyendo que el tratamiento a base de desbridamiento más la colocación local de antibiótico, o el uso de terapia laser tiene mejores resultados en disminuir los signos de la inflamación que la debridación, más el uso de clorhexidina.

Las terapias a base de laser han tenido cierto soporte en la literatura, el diodo a $810 \mathrm{~nm}$ tiene un efecto bactericida debido al aumento de la temperatura que presenta, esto no daña la superficie del implante y este efecto térmico que se genera debilita la adhesión del cálculo a la superficie del implante (Roncati et al., 2013), por otra parte Romanos et al. (2006) reportaron que el uso de laser de dióxido de carbono tiene un efecto en la inducción de la unión de los osteoblastos así como en estimular la formación de hueso cuando este se usa en superficies de titanio, lo cual se confirma con el estudio realizado por Javed et al. (2013) en donde se destaca que el uso de este laser reduce también el daño a los tejidos ocasionado por las altas temperaturas que manejan otros tipos de láser, Natto et al. (2015) mencionan que el láser Er: Yag y el de dióxido de carbono son los que cuentan con mayor evidencia en el tratamiento de la periimplantitis 6 meses posteriores al mismo.

Otra parte importante del tratamiento no solo se enfoca a eliminar la etiología de la periimplantitis si no que incluye también la regeneración del tejido periimplantario, esto mediante el uso de membranas de colágeno, factores de crecimiento entre otros. Debido al daño que se presenta en los tejidos periimplantarios ocasionados por el proceso inflamatorio, se disminuye considerablemente la regeneración vascular, Mierzwinska-Nastalska et al. (2010) midieron la presencia del factor de crecimiento endotelial vascular (VEGF) en el líquido crevicular reportando que en zonas periféricas a la periimplantitis se encuentra una mayor concentración del mismo. Froum et al. (2012) realizaron un trabajo en el cual trataron a 31 pacientes de periimplantitis bajo el protocolo de curetaje abierto, desinfección del implante, colocación de matriz derivada de esmalte, aloinjerto y hueso mineralizado, obteniendo resultados favorables en tejido blando en comparación con las mediciones iniciales realizadas, llegando a la conclusión que el tratamiento de la periimplantitis debe complementarse con diferentes tipos de procedimientos como el curetaje, desinfección de la superficie del implante y regeneración.

Como ya se había mencionado en el caso de que el paciente desarrolle mucositis periimplantaria la opción de tratamiento es mejorar las condiciones de higiene bucal, enjuagues con antisépticos por 15 días con clorhexidina al $0,12 \%$ y en caso de no remitir la inflamación se complementa con tratamiento sistémico a base de amoxicilina con ácido clavulánico o clindamicina. Cuando se presenta la periimplantitis el tratamiento lo enfocamos a la eliminación del cálculo realizando curetaje abierto, desinfección del implante con lavado de la superficie del mismo con clorhexidina al $0,12 \%$ y en casos específicos de que el defecto óseo ponga en peligro la estabilidad de la rehabilitación se emplean terapias de regeneración con la aplicación de hueso liofilizado y membrana de colágeno, todo lo anterior reforzado con una adecuada técnica de higiene.

\section{CONCLUSIONES}

La colocación de implantes dentales hoy en día es una opción de rehabilitación muy utilizada que devuelve tanto la funcionalidad y la estética que se ve disipada al perder los órganos dentarios, sin embargo no todos los pacientes son candidatos de colocar este tipo de aditamentos no solo por la presencia tanto de la calidad como cantidad de hueso presente si no por las condiciones tanto locales como sistémicas de salud del paciente, por lo que el realizar una adecuada valoración, control y seguimiento de los pacientes con implantes se vuelve fundamental para el éxito de los mismos.

GARCÍA-CALDERÓN, A. G.; DONOHUE-CORNEJO, A.; CUEVAS-GONZÁLEZ, M. V.; ÁVILA-VALDÉZ, R. \& CUEVAS-GONZÁLEZ, J. C. Peri-implantitis; literature review. Int. J. Odontostomat., 10(2) :255-260, 2016.

ABSTRACT: The use of implants has taken a giant leap in recent years, however, greater demand has also resulted in an increase in the failure of these procedures. There are multiple reasons involved such as; deficiencies in 
the amount and quality of bone, preexisting bone disease, poor surgical technique, improper implant, tobacco habit, among others. The peri-implant mucositis and peri-implantitis are the main causes of complications in implants, patients with chronic degenerative diseases, smokers and patients with poor oral hygiene are considered risk patients to present peri-implantitis. The literature provides surgical and nonsurgical treatments which are focused on the elimination of microorganisms and the disinfection of the implant surface; surgical treatment as debridement and curettage are designed to remove plaque, disinfect the implant surface and remove damaged tissue caused by the inflammatory process, the performance of an adequate medical history and prior assessment will identify patients with greater or lesser risk of developing peri-implantitis, maintenance appointments help in the early detection of possible complications.

KEY WORDS: peri-implantitis, peri-implant mucositis, periodontal disease.

\section{REFERENCIAS BIBLIOGRÁFICAS}

Ata-Ali, J.; Candel-Marti, M. E.; Flichy-Fernández, A. J.; Peñarrocha-Oltra, D.; Balaguer-Martinez, J. F. \& Peñarrocha Diago, M. Peri-implantitis: associated microbiota and treatment. Med. Oral Patol. Oral Cir. Bucal, 16(7):937-43, 2011.

Baig, M. R. \& Rajan, M. Effects of smoking on the outcome of implant treatment: a literature review. Indian J. Dent. Res., 18(4):190-5, 2007.

Candel-Martí, M. E.; Flichy-Fernández, A. J.; Alegre-Domingo, T.; Ata-Ali, J. \& Peñarrocha-Diago, M. A. Interleukins IL-6, IL-8, IL-10, IL-12 and periimplant disease. An update. Med. Oral Patol. Oral Cir. Bucal, 16(4):e518-21, 2011.

Casado, P. L.; Pereira, M. C.; Duarte, M. E. \& Granjeiro, J. M. History of chronic periodontitis is a high risk indicator for peri-implant disease. Braz. Dent. J., 24(2):136-41, 2013.

Cavalli, N.; Corbella, S.; Taschieri, S. \& Francetti, L. Prevalence of peri-implant mucositis and peri-implantitis in patients treated with a combination of axial and tilted implants supporting a complete fixed denture. Sci. World J., 2015:874842, 2015.

Dhir, S. Biofilm and dental implant: The microbial link. J. Indian Soc. Periodontol., 17(1):5-11, 2013.

Ferreira, S. D.; Silva, G. L.; Cortelli, J. R.; Costa, J. E. \& Costa, F. O. Prevalence and risk variables for peri-implant disease in Brazilian subjects. J. Clin. Periodontol., 33(12):929-35, 2006.
Froum, S. J.; Froum, S. H. \& Rosen, P. S. Successful management of peri-implantitis with a regenerative approach: a consecutive series of 51 treated implants with 3- to 7.5-year follow-up. Int. J. Periodontics Restorative Dent., 32(1):11-20, 2012.

García-Calderón, M.; Cabezas Talavero, J.; Gallego Romero, D. \& Torres Lagares, D. Diagnóstico y tratamiento de las periimplantitis. Actualización en el diagnóstico clínico y en el tratamiento de las periimplantitis. Av. Periodoncia, 16(1):9-18, 2004.

Javed, F.; Hussain, H. A. \& Romanos, G. E. Re-stability of dental implants following treatment of peri-implantitis. Interv. Med. Appl. Sci., 5(3):116-21, 2013.

Kadkhodazadeh, M. \& Amid, R. A New Classification for the relationship between periodontal, periapical, and periimplant complications. Iran Endod. J., 8(3):103-8, 2013.

Kasat, V. \& Ladda, R. Smoking and dental implants. J. Int. Soc. Prev. Community Dent., 2(2):38-41, 2012.

Maruyama, N.; Maruyama, F.; Takeuchi, Y.; Aikawa, C.; Izumi, Y. \& Nakagawa, I. Intraindividual variation in core microbiota in peri-implantitis and periodontitis. Sci. Rep., 4:6602, 2014.

McCrea, S. J. Advanced peri-implantitis cases with radical surgical treatment. J. Periodontal. Implant. Sci., 44(1):3947, 2014.

Mierzwinska-Nastalska, E.; Lomzynski, L., JaworskaZaremba, M. \& Kostrzewa-Janicka, J. Vascular endothelial growth factor in gingival crevicular fluid around dental implants. Eur. J. Med. Res., 15 (Suppl. 2):88-91, 2010.

Mohamed, J. B.; Alam, M. N.; Singh, G. \& Chandrasekaran, S. C. The management of retrograde peri-implantitis: a case report. J. Clin. Diagn. Res., 6(9):1600-2, 2012.

Muthukuru, M.; Zainvi, A.; Esplugues, E. O. \& Flemmig, T. F. Non-surgical therapy for the management of periimplantitis: a systematic review. Clin. Oral Implants Res., 23 (Suppl. 6):77-83, 2012.

Natto, Z. S.; Aladmawy, M.; Levi, P. A. Jr. \& Wang, H. L. Comparison of the efficacy of different types of lasers for the treatment of peri-implantitis: a systematic review. Int. J. Oral Maxillofac. Implants, 30(2):338-45, 2015.

Pedrazzi, V.; Escobar, E. C.; Cortelli, J. R.; Haas, A. N.; Andrade, A. K.; Pannuti, C. M.; Almeida, E. R.; Costa, F. O.; Cortelli, S. C. \& Rode, S. M. Antimicrobial mouthrinse use as an adjunct method in peri-implant biofilm control. Braz. Oral Res., 28 Spec. No. pii:S1806$83242014000200301,2014$. 
Prathapachandran, J. \& Suresh, N. Management of periimplantitis. Dent. Res. J. (Isfahan), 9(5):516-21, 2012.

Real-Osuna, J.; Almendros-Marqués, N \& Gay-Escoda, C. Prevalence of complications after the oral rehabilitation with implant-supported hybrid prostheses. Med. Oral Patol. Oral Cir. Bucal, 17(1):e116-21, 2012.

Rodríguez-Ortega, L.; Velasco-Ortega, V.; CastellanosCosano, L.; Martín-González, J.; López-Frías, F. J. \& Segura-Egea, J. J. Patología implanto-endodóncica: concepto, tipos, diagnóstico, tratamiento y prevención. Av. Periodoncia, 25(2):83-90, 2013.

Romanos, G.; Crespi, R.; Barone, A. \& Covani, U. Osteoblast attachment on titanium disks after laser irradiation. Int. J. Oral Maxillofac. Implants, 21(2):232-6, 2006.

Roncati, M.; Lucchese, A. \& Carinci, F. Non-surgical treatment of peri-implantitis with the adjunctive use of an 810-nm diode laser. J. Indian Soc. Periodontol., 17(6):812-5, 2013.

Sánchez-Gárces, M. A. \& Gay-Escoda, C. Periimplantitis. Med. Oral Patol. Oral Cir. Bucal, 9 Suppl.:69-74; 63-9, 2004.

Schmidlin, P. R.; Sahrmann, P.; Ramel, C.; Imfeld, T.; Müller, J.; Roos, M. \& Jung, R. E. Peri-implantitis prevalence and treatment in implant-oriented private practices: a cross-sectional postal and Internet survey. Schweiz Monatsschr Zahnmed, 122(12):1136-44, 2012.

Segura Andrés, G.; Gil-Pulido, R.; Vicente González, F.; Ferreiroa Navarro, A.; Faus López, J. \& Agustín Panadero, R. Periimplantitis y mucositis periimplantaria. Factores de riesgo, diagnóstico y tratamiento. Av. Periodoncia, 27(1):25-36, 2015.

Smeets, R.; Henningsen, A.; Jung, O.; Heiland, M.; Hammächer, C. \& Stein, J. M. Definition, etiology, prevention and treatment of peri-implantitis--a review. Head Face Med., 10:34, 2014.

Taschieri, S.; Weinstein, R.; Del Fabbro, M. \& Corbella, S. Erythritol-enriched air-polishing powder for the surgical treatment of peri-implantitis. Sci. World J., 2015:802310, 2015.

Valderrama, P.; Blansett, J. A.; Gonzalez, M. G.; Cantu, M. G. \& Wilson, T. G. Detoxification of implant surfaces affected by peri-implant disease: An overview of nonsurgical methods. Open Dent. J., 8:77-84, 2014.

Yaghobee, S.; Khorsand, A.; Rasouli Ghohroudi, A. A.; Sanjari, K. \& Kadkhodazadeh, M. Assessment of interleukin-1beta and interleukin-6 in the crevicular fluid around healthy implants, implants with peri-implantitis, and healthy teeth: a cross-sectional study. J. Korean Assoc. Oral Maxillofac. Surg., 40(5):220-4, 2014.
Dirección de Correspondencia:

Dr. Juan Carlos Cuevas González

Departamento de Estomatología

Universidad Autónoma de Ciudad Juárez

Anillo Envolvente del Pronaf $s / n$,

Zona Pronaf, 32315 Cd Juárez

Chihuahua

MÉXICO

Email: cuevas_gonzalez@hotmail.com

Recibido : 23-11-2015

Aceptado: 26-04-2016 